\title{
Agresión entre pares y bienestar psicológico en estudiantes de una universidad pública de Lima Metropolitana
}

\author{
Bienestar agresion peer and psychological among students of a \\ public university of Lima \\ Carlos Velásquez C 1 ., William Montgomery U., Alejandro Dioses C., Ricardo Pomalaya V., \\ José Chávez Z., Kevin Egúsquiza V., Victoria Cavero H., Corina Wong Ch. \\ Universidad Nacional Mayor de San Marcos, Lima, Perú \\ InStituto de InVESTIGACIONES PsicológICA \\ (RECIBIDO 03-09 2014, ACEPTADO 02-12-2014)
}

\begin{abstract}
RESUMEN
La presente investigación examina las relaciones entre la agresión entre pares (bullying) y el bienestar psicológico, en estudiantes de las diferentes áreas profesionales de la Universidad de San Marcos: Ciencias de la Salud, Humanidades, Ciencias Sociales, Ciencias Básicas, Ingenierías y Económica Empresarial. Para ello, se relaciona la Escala de Violencia entre Pares de Espelage (actitud hacia la violencia, conductas prosociales, conducta agresiva y ciberacoso) con la Escala de Bienestar Psicológico de Ryff (relaciones positivas, autonomía, dominio de entorno, crecimiento personal y propósito en la vida). El estudio es de tipo descriptivo-correlacional, utilizando un muestreo no probabilístico de la población considerada. Los instrumentos en mención fueron analizados psicométricamente para adaptarlos a nuestra realidad. Como resultados se encontró que las conductas solidarias se vinculan con la aceptación de sí mismos, el aprovechamiento de sus capacidades y la claridad en las metas personales; por el contrario, la actitud favorable hacia las conductas agresivas se vincula con una baja aceptación de sí mismo y un inadecuado manejo de las oportunidades que ofrece el medio. Asimismo, se registraron diferencias respecto a la edad, al género y a las áreas profesionales.
\end{abstract}

Palabras clave: Bullying, agresión entre pares, bienestar, conducta pro social, violencia.

1 E-mail: cvelasquezcenteno@hotmail.com 


\begin{abstract}
This research examines the relationships between peer aggression (bullying) and psychological well-being in students from different professional areas of San Marcos University: Health Sciences, Humanities, Social Sciences, Basic Sciences, Engineering and Economic Enterprise. To do this, Scale Peer Violence relates to Espelage (attitude toward violence, prosocial behavior, aggressive behavior and cyberbullying) with Scale of Ryff Psychological Well-Being (positive relationships, autonomy, mastery of environment, personal growth and purpose in life). The study is descriptive-correlational, using an non-probability sampling of the population considered. Instruments mentioned were psychometrically analyzed to fit our reality. As a result it was found that supportive behaviors are linked to the acceptance of itself, use skills and clarity of personal goals; on the contrary, the favorable attitude toward aggressive behavior is associated with low self-acceptance and the mismanagement of the opportunities offered by the environment. Finally, differences were found in age, gender and professional areas.
\end{abstract}

Keywords: Bullying, aggression between pairs, psychological well-being, prosocial behavior, violence.

\title{
INTRODUCCIÓN
}

La investigación sobre bullying, agresión entre pares o acoso escolar se inició en la década del 50; concretándose como la intimidación casi permanente de un individuo sobre otro, y afectando a la víctima, al agresor y a los espectadores de tales episodios. Su incidencia daña las relaciones y aprendizajes sociales, en tanto es un referente de conducta imitable o de habituación hacia el sufrimiento ajeno, motivando en ocasiones ansiedad o culpa (Quintana, Montgomery y Malaver, 2009). La agresión entre pares en el ámbito educativo, independientemente del nivel, es un problema de alta incidencia y con características alarmantes en la sociedad peruana (MINEDU, 2010). Así, uno de sus rasgos distintivos es la poca atención que recibe, tanto por parte de los padres, profesores o autoridades escolares; lo que conduce al joven a un estado de desesperanza, que en el mejor de los casos, lo lleva a abandonar la institución educativa. Es de esta manera que el perfil de los individuos que ejercen este tipo de violencia es de interés para los investigadores y profesionales del comportamiento, en tanto puede ofrecer pistas para ampliar el panorama sobre el problema y articular estrategias de prevención.

Una de las interrogantes sobre el perfil del agresor en episodios de bullying es la de su relación con el bienestar psicológico, un concepto ligado tanto a la percepción subjetiva que se tiene respecto a los logros conseguidos, como al grado de satisfacción personal con sus acciones pasadas, presentes y futuras (Diener, Suh, Lucas y Smith, 1999). ¿Hay, en este sentido, algún tipo de característica saltante en dicha relación tal como existe, por ejemplo, entre la resiliencia y el ejercicio del acoso escolar? Parece ser que, debido a su ligazón con los modos de afrontamiento al estrés, el comportamiento resiliente no es compatible con el ejercicio del bullying. Por tanto, cabría esperar que el bienestar psicológico, uno de los 
componentes del comportamiento resiliente, tampoco lo fuera (Marqueza, 2005). De acuerdo con las revisiones realizadas sobre la percepción de la violencia de alumnos, docentes y administradores del sistema educativo en Uruguay (Viscardi, 2003), Colombia (Castrillon y Vieco, 2002; Paredes, Álvarez y Vernón, 2008), y en Chile (Trauttman, 2008), los factores del bienestar psicológico pueden ser afectados por la agresión entre pares. Por ello, la presente investigación desea profundizar al respecto.

El bienestar psicológico es una variable de amplio acceso (Salanova y Martínez, 2005). En anterior indagación, Velásquez, Montgomery, Montero, Pomalaya, Dioses, Velásquez, Araki y Reynoso (2008), abordaron empírica y conceptualmente la problemática de las interacciones entre las variables de bienestar psicológico, asertividad y rendimiento académico en estudiantes de facultades representativas de las diversas áreas de estudio de la Universidad de San Marcos: Medicina (Ciencias de la Salud), Derecho y Educación (Humanidades y Ciencias Sociales), Matemáticas (Ciencias Básicas), Ingeniería Industrial (Ingenierías), y Administración (Económica Empresarial); utilizando la Escala de Bienestar Psicológico de Ryff que evalúa factores como relaciones positivas, autonomia, dominio de entorno, crecimiento personal y proposito en la vida, y encontrando que tiene una buena confiabilidad y utilidad para la medición de dicha variable. Por lo tanto, nuevamente parte de este grupo de investigación utiliza dicho instrumento para este estudio.

En base a las consideraciones reseñadas, el estudio pretende cumplimentar una serie de objetivos que serán de suma importancia: a) determinar la relación entre la agresión entre pares y el bienestar psicológico de los estudiantes de las diferentes áreas profesionales de una universidad pública de lima metropolitana; b) describir las características de la agresión entre pares en función a la edad, sexo y áreas profesionales; c) describir las características del bienestar psicológico en función a la edad, sexo y área profesional; d) determinar la relación entre las áreas de la agresión entre pares y el bienestar psicológico en función a la edad, sexo y áreas profesionales; y e) determinar las diferencias entre las áreas de la agresión entre pares y el bienestar psicológico en función a la edad, sexo y áreas profesionales. Asimismo, se considera importante adaptar las escalas de agresión entre pares y de bienestar psicológico empleadas en la presente investigación a la muestra universitaria limeña, con el fin de contar con instrumentos adaptados y estandarizados para mejorar el proceso de evaluación y diagnóstico de dichas variables y para el desarrollo de programas, estrategias y capacitaciones dirigidas a tal población.

\section{METODOLOGÍA}

\section{Muestra}

Conformada por 1168 alumnos, de los cuales 675 son del sexo masculino y 493 del sexo femenino correspondientes a seis áreas profesionales: Ciencias de la Salud, Humanidades, Ciencias Sociales, Ciencias Básica, Ingeniería y EconómicoEmpresariales (tabla 1). 
Tabla 1. Distribución de la muestra por áreas profesionales y género

\begin{tabular}{lccc}
\hline \multirow{2}{*}{ AREAS } & \multicolumn{2}{c}{ Género } & \multirow{2}{*}{ Total } \\
\cline { 2 - 3 } & Masculino & Femenino & \\
\hline Ciencias de la Salud & 33 & 68 & 101 \\
Humanidades & 135 & 107 & 242 \\
Ciencias Sociales & 103 & 98 & 201 \\
Ciencias Básicas & 91 & 59 & 150 \\
Ingenierías & 208 & 66 & 274 \\
Económico-Empresariales & 105 & 95 & 200 \\
\hline Total & 675 & 493 & 1168 \\
\hline
\end{tabular}

\section{Diseño}

De tipo Descriptivo-Correlacional.

\section{Instrumentos}

La agresión entre pares (bullying), se evaluó con la Escala de Agresión entre Pares de Espelage \& Holt, (2001) y Espelaje, Mebane \& Swearer, (2004), la cual fue adaptada para la población sanmarquina (Anexo $\mathrm{N}^{\circ} 01$ ), manteniendo las sub escalas originales: La sub escala influencia externa, que evalúa cómo influye en las conductas de agresión de los jóvenes el comportamiento de los pares, y las actitudes de los adultos con los cuales ellos comparten la mayor parte del tiempo; la sub escala actitud hacia la violencias, que evalúa la actitud personal de los estudiantes con respecto a las conductas agresivas, al acto físico de pelear y a modos alternativos de resolver conflictos; la sub escala de conductas prosociales, que evalúa las conductas de solidaridad con los compañeros; la sub escala de conductas agresivas, que investiga la frecuencia con que los encuestados incurren en comportamientos de agresión entre pares; la sub escala de ciberacoso, que indaga las diferentes modalidades en que se expresa el acoso virtual; la escala está conformada por 44 ítems, cuyas respuestas son de tipo Likert, del 1 al 5, donde 1 es ninguno y 5 es todos. La validez se realizó a través del ítem-test con una $\mathrm{r}$ entre .203 y .722, y la confiabilidad obtenida a través del Alfa de Crombach fluctuó entre .534 y .767.

El bienestar psicológico, por su parte, se evaluó utilizando la Escala de Bienestar Psicológico de Ryff. Ryff, 1985; Ryff \& Keyes, 1995). Esta escala fue adaptada para la población objetivo del presente estudio (Anexo 2), manteniendo en sus áreas la misma estructura original: Autoaceptación, en la que las personas con una alta autoaceptación tienen una actitud positiva hacia sí mismas, aceptan los diversos aspectos de su personalidad, incluyendo los negativos, y se sienten bien respecto a su pasado; relaciones positivas, que consiste en tener relaciones de calidad con los demás, gente con la que se pueda contar, alguien a quien amar; Autonomía, que consiste en tener la sensación de que uno puede elegir, tomar tus propias decisiones para sí y para su vida, incluso si van en contra de la opinión 
mayoritaria, manteniendo la independencia personal y las convicciones; dominio del entorno, que hace referencia al manejo de las exigencias y oportunidades de tu ambiente para satisfacer tus necesidades y capacidades; crecimiento personal, que consiste en sacar el mayor partido a los talentos y habilidades, utilizando todas las capacidades, desarrollar las potencialidades y seguir creciendo como persona; y finalmente, propósito en la vida, referido a que la vida tenga un sentido y un propósito. La escala cuenta con 29 ítems, y sus áreas están compuestas por $4 \mathrm{o}$ 9 ítems, con respuestas de tipo Likert del 1 al 6, donde 1 es totalmente en desacuerdo y 6 es totalmente de acuerdo. La validez se estimó a través del método del ítem-test, obteniéndose una $\mathrm{r}$ entre .207 y .554 , y la confiabilidad obtenida a través del Alfa de Crombach fluctuó entre .748 y .901.

\section{Procedimiento}

El estudio se llevó a cabo en diversas facultades de las áreas de estudio que ofrece la Universidad Nacional Mayor de San Marcos, previa coordinación con las autoridades, administrando los instrumentos intencionalmente a los alumnos presentes en los diferentes ciclos (iniciales, intermedios y avanzados) a través de un muestreo accidental. La aplicación de las pruebas se realizó dentro del salón de clases.

\section{RESULTADOS}

Como puede observarse en la tabla 2, se esboza la agresión entre pares en función a la edad en rangos (adolescentes de 16 a 18 años, los jóvenes de 19 a 25 años y adultos de 26 a 50 años) no es significativa, por lo que se concluye que no guardan relación con ningún componente.

Tabla 2. Relación entre la agresión entre pares y la edad

\begin{tabular}{c|c|c|c|c|c}
\hline \multicolumn{6}{c}{ Estadísticos de contraste } \\
\hline & $\begin{array}{c}\text { INF_ } \\
\text { EXTERNA }\end{array}$ & $\begin{array}{c}\text { ACT_ } \\
\text { VIOLENCIA }\end{array}$ & C_PROSOCIALES & C_AGRESIVAS & CIBERACOSO \\
\hline $\begin{array}{c}\text { Chi- } \\
\text { cuadrado } \\
\text { Gl }\end{array}$ & .143 & .548 & 1.852 & 3.833 & 3.262 \\
$\begin{array}{c}\text { Sig. } \\
\text { asintót. }\end{array}$ & 2 & 2 & 2 & 2 & 2 \\
\hline \multicolumn{6}{c}{\begin{tabular}{c} 
Prueba de Kruskal-Wallis \\
\multicolumn{6}{c}{ Variable de agrupación: Edad 2 }
\end{tabular}} \\
\hline
\end{tabular}

En cuanto a la agresión entre pares y el género, puede observarse en la tabla 3 que existen diferencias significativas en todas las subescalas, por lo que se puede concluir que sí existen diferencias entre el género masculino y femenino, siendo el género masculino más agresivo que el femenino; salvo en la subescala de conductas prosociales, donde el género femenino presenta mayor puntuación, por lo que se infiere que son más solidarias con los compañeros que el género masculino. 
Tabla 3. Relación entre la agresión entre pares y el género.

\begin{tabular}{c|c|c|c|c|c}
\hline \multicolumn{7}{c|}{ Estadísticos de contraste } \\
\hline & $\begin{array}{c}\text { INF_- } \\
\text { EXTERNA }\end{array}$ & $\begin{array}{c}\text { ACT_- } \\
\text { VIOLENCIA }\end{array}$ & C_PROSOCIALES & $\begin{array}{c}\text { C_- }_{-} \\
\text {AGRESIVAS }\end{array}$ & CIBERACOSO \\
\hline $\begin{array}{c}\text { U de Mann- } \\
\text { Whitney }\end{array}$ & 102967.00 & 102446.000 & 135783.500 & 118172.500 & 119391.5 \\
W de Wilconox & 223753.00 & 224217.000 & 363933.500 & 238958 & 241162.5 \\
Z & -11.006 & -11.303 & -5.397 & -8.359 & -8.902 \\
$\begin{array}{c}\text { Sig. asintót. } \\
\text { (bilateral) }\end{array}$ & .000 & .000 & .000 & .000 & .000 \\
\hline \multicolumn{7}{c}{ Variable de agrupación: Sexo } \\
\hline
\end{tabular}

Por otro lado, al relacionar la agresión entre pares con las áreas profesionales (tabla 4), se ve que no hay diferencias en las subescalas de influencia externa, actitud hacia la violencia y conductas agresivas. Al contrario, sí existen diferencias en las subescalas de conductas pro sociales y de ciberacoso, donde las ciencias de la salud e ingenierías tienen mayor rango promedio, lo que indica que en el área de ciencias de la salud son más solidarios con los compañeros, mientras que en las ingenierías, existe agresión virtual.

Tabla 4. Relación entre la agresión entre pares y las áreas profesionales.

\begin{tabular}{c|c|c|c|c|c}
\hline \multicolumn{7}{c}{ Estadísticos de contraste } \\
\hline & $\begin{array}{c}\text { INF_ } \\
\text { EXTERNA }\end{array}$ & $\begin{array}{c}\text { ACT_ } \\
\text { VIOLENCIA }\end{array}$ & C_PROSOCIALES & $\begin{array}{c}\text { C_- }_{-} \\
\text {AGRESIVAS }\end{array}$ & CIBERACOSO \\
\hline $\begin{array}{c}\text { Chi- } \\
\text { cuadrado } \\
\text { Gl }\end{array}$ & 8.152 & 10.076 & 17.815 & 7.610 & 18.397 \\
Sig. asintót. & 5 & 5 & 5 & 5 & 5 \\
\hline \multicolumn{6}{c}{$\begin{array}{c}\text { Prueba de Kruskal-Wallis } \\
\text { Variable de agrupación: Edad2 }\end{array}$} \\
\hline
\end{tabular}

Al observar la tabla 5, donde se relaciona el bienestar psicológico con la edad expresada en rangos, no existen diferencias significativas a excepción de autonomía, en la cual los adolescentes tienen mayor promedio, lo que señala que son capaces de tomar sus propias decisiones y mantener su independencia personal.

Tabla 5. Relación entre las áreas del bienestar psicológico y la edad.

\begin{tabular}{|c|c|c|c|c|c|c|}
\hline \multicolumn{7}{|c|}{ Estadísticos de contraste } \\
\hline & AUTOACEPTACIÓN & $\begin{array}{l}\mathrm{RE}_{-} \\
\mathrm{POST}\end{array}$ & AUTONOMÍA & $\begin{array}{c}\text { DOM }_{-} \\
\text {ENTORNO }\end{array}$ & $\begin{array}{c}\text { CRECI } \\
\text { PERSONAL }\end{array}$ & $\begin{array}{l}\text { PROPÓSITO } \\
\text { VIDA }\end{array}$ \\
\hline Chi-cuadrado & 2.284 & 1.439 & 7.821 & 3.924 & 1.976 & 1.455 \\
\hline Gl & 2 & 2 & 2 & 2 & 2 & 2 \\
\hline Sig. asintót. & .319 & .487 & .020 & .141 & .372 & .483 \\
\hline \multicolumn{7}{|c|}{$\begin{array}{c}\text { Prueba de Kruskal-Wallis } \\
\text { Variable de agrupación: Edad2 }\end{array}$} \\
\hline
\end{tabular}


En cuanto a la relación entre el bienestar psicológico y el género, se observa en la tabla 6 que éste último no influye ni está relacionado con el bienestar psicológico, a excepción del componente de propósito de vida, en el cual las mujeres tienen mayor rango promedio, del cual se puede inferir que el género femenino define claramente una serie de objetivos que les permiten dotar a su vida de sentido.

Tabla 6. Relación entre las áreas del Bienestar Psicológico y el género.

\begin{tabular}{c|c|c|c|c|c|c}
\hline \multicolumn{7}{c}{ Estadísticos de contraste } \\
\hline & AUTOACEPTACIÓN & RE_POST & AUTONOMÍA & $\begin{array}{c}\text { DOM_- } \\
\text { ENTORNO }\end{array}$ & $\begin{array}{c}\text { CRECI_ } \\
\text { PERSONAL }\end{array}$ & $\begin{array}{c}\text { PROPÓSITO_ } \\
\text { VIDA }\end{array}$ \\
\hline $\begin{array}{c}\text { U de Mann- } \\
\text { Whitney } \\
\text { W de }\end{array}$ & 165161.50 & 155606.5 & 158611.500 & 165131.000 & 157412.000 & 150151.500 \\
$\begin{array}{c}\text { Wilcoxon } \\
\text { Z }\end{array}$ & 391962.500 & 277377.50 & 386761.500 & 393281.000 & 385562.000 & 377626.500 \\
$\begin{array}{c}\text { Sig. asintót. } \\
\text { (bilateral) }\end{array}$ & -.130 & -1.898 & -1.311 & -.162 & -1.582 & -2.709 \\
\hline \multicolumn{7}{c}{ Prueba de Kruskal-Wallis } \\
\hline
\end{tabular}

En la tabla 7 se observa al relacionar el bienestar psicológico con las áreas profesionales que sí están relacionadas, y en casi todos los componentes el área de salud tiene mayor rango promedio, por lo que puede señalarse que los participantes de las ciencias de la salud se caracterizan por aceptar diversos aspectos de su personalidad con actitud positiva, por el manejo de exigencias y oportunidades del ambiente, por desarrollar sus habilidades utilizando al máximo sus capacidades, y por definir sus objetivos con visión. Por otro lado, el área económico-empresarial se caracteriza por tener relaciones de calidad con los demás y la rápida toma de decisiones al margen de la influencia externa.

Tabla 7. Relación entre las áreas del bienestar psicológico y las áreas profesionales.

\begin{tabular}{|c|c|c|c|c|c|c|}
\hline \multicolumn{7}{|c|}{ Estadísticos de contraste } \\
\hline & AUTOACEPTACIÓN & $\begin{array}{c}\mathrm{RE}_{-} \\
\text {POST }\end{array}$ & AUTONOMÍA & $\begin{array}{c}\text { DOM- } \\
\text { ENTORNO }\end{array}$ & $\begin{array}{l}\text { CRECI } \\
\text { PERSONAL }\end{array}$ & $\begin{array}{c}\text { PROPÓSITO_ } \\
\text { VIDA }\end{array}$ \\
\hline $\begin{array}{l}\text { Chi- } \\
\text { cuadrado }\end{array}$ & 29.935 & 47.711 & 64.443 & 50.614 & 29.821 & 19.651 \\
\hline Gl & 5 & 5 & 5 & 5 & 5 & 5 \\
\hline $\begin{array}{l}\text { Sig. } \\
\text { asintót. }\end{array}$ & .000 & .000 & .000 & .000 & .000 & .001 \\
\hline \multicolumn{7}{|c|}{ Prueba de Kruskal-Wallis } \\
\hline
\end{tabular}

$\mathrm{Al}$ observar la tabla 8 , puede afirmarse que casi todas las correlaciones son altamente significativas $\mathrm{p}<0.05$ y $\mathrm{p}<0.01$ pero con valores de correlación bajas. Para efectos de la interpretación, se consideran significativos solo las correlaciones con $\mathrm{p}<0.05$ y coeficiente de correlación mayores de 0.2 . En ese sentido, las subescalas de influencia externa y conductas prosociales correlacionan significativamente 
con todas las áreas del bienestar psicológico, a excepción del área de autonomía. La subescala de influencia externa, se correlaciona positivamente con el área de relaciones positivas determinando esto que a mayor calidad de las relaciones con los demás es mayor la influencia que se tiene sobre las conductas de agresión de los pares. La subescala de influencia externa se correlaciona negativamente con las áreas de autoaceptación, dominio del entorno, crecimiento personal y propósito de vida, con lo que puede concluirse que a mayor influencia externa existe una menor actitud positiva hacia sí mismo, un menor manejo de la exigencias y oportunidades, poco aprovechamiento de las habilidades y poca claridad en la definición de metas.

Por otro lado, la subescala de conductas prosociales, se correlaciona positivamente con las áreas de autoaceptación, dominio del entorno, crecimiento personal y propósito de vida, lo que indicaría que cuan más solidario se es hay una mayor aceptación de sí mismo, así como un buen manejo de las exigencias y oportunidades del ambiente, un buen aprovechamiento de sus potencialidad y una definición de sus metas. Del mismo modo la subescala de conductas pro sociales se correlaciona negativamente con el área de relaciones positivas determinando esto que a mayor solidaridad con los pares menor es la calidad de la relación.

Tabla 8. Correlación entre las áreas de la agresión entre pares y el bienestar psicológico.

\begin{tabular}{cccccc}
\hline \multicolumn{5}{c}{ Correlaciones todos los sujetos } & \\
\hline & INF_ & ACT_ & C_PRO_ & C_- & CIBERACOSO \\
& EXTERNA & VIOLENCIA & SOCIALES & AGRESIVAS & \\
\hline AUTOACEPTACIÓN & -0.201 & -0.165 & 0.313 & -0.088 & -0.090 \\
& 0.000 & 0.000 & 0.000 & 0.003 & 0.002 \\
& 1162.000 & 1166.000 & 1166.000 & 1163.000 & 1166.000 \\
RE_POST & 0.247 & 0.183 & -0.320 & 0.116 & 0.147 \\
& 0.000 & 0.000 & 0.000 & 0.000 & 0.000 \\
AUTONOMÍA & 1164.000 & 1168.000 & 1168.000 & 1165.000 & 1168.000 \\
& 0.099 & 0.162 & -0.175 & 0.032 & 0.090 \\
& 0.001 & 0.000 & 0.000 & 0.283 & 0.002 \\
DOM_ENTORNO & 1163.000 & 1167.000 & 1167.000 & 1164.000 & 1167.000 \\
& -0.221 & -0.172 & 0.248 & -0.073 & -0.112 \\
& 0.000 & 0.000 & 0.000 & 0.012 & 0.000 \\
& 1163.000 & 1167.000 & 1167.000 & 1165.000 & 1167.000 \\
CRECI_PERSONAL & -0.217 & -0.155 & 0.322 & -0.075 & -0.104 \\
& 0.000 & 0.000 & 0.000 & 0.011 & 0.000 \\
& 1164.000 & 1168.000 & 1168.000 & 1165.000 & 1168.000 \\
& -0.209 & -0.114 & 0.290 & -0.102 & -0.106 \\
& 0.000 & 0.000 & 0.000 & 0.001 & 0.000 \\
& 1161.000 & 1165.000 & 1165.000 & 1162.000 & 1165.000 \\
\hline
\end{tabular}


En relación al grupo etario conformado por adolescentes (tabla 9), existe una correlación negativa entre la subescala de influencia externa y las áreas de autoaceptación, dominio del entorno, crecimiento personal y propósito de vida; lo cual indicaría que ante una mayor influencia que los adolescentes tienen sobre las conductas agresivas en los pares menor es la aceptación de sus diversos aspectos de personalidad, el manejo de las exigencias y oportunidades del ambiente, el aprovechamiento de sus habilidades y la claridad con la que definen sus metas.

Por otro lado, existe una correlación positiva entre la subescala conductas prosociales y las áreas de autoaceptación, dominio del entorno y propósito de vida, a partir de lo cual se puede inferir que a mayor conductas solidarias con sus pares mayor es la aceptación de sí mismo, el aprovechamiento de sus potencialidades y la claridad en la definición de sus metas. Asimismo, dicha subescala se correlaciona negativamente con las áreas de relaciones positivas y dominio del entorno, por lo cual se infiere que a mayor ratio de conductas solidarias menor es la calidad de las relaciones que se sostienen con los demás, así como la capacidad para tomar decisiones de manera independiente.

Tabla 9. Correlación entre las subescalas de la agresión entre pares y las áreas del bienestar Psicológico en función a la edad. Adolescentes.

\begin{tabular}{|c|c|c|c|c|c|}
\hline \multicolumn{6}{|c|}{ Correlaciones según edad en adolescentes } \\
\hline & $\begin{array}{c}\text { INF_- }_{-} \\
\text {EXTERNA }\end{array}$ & $\begin{array}{c}\text { ACT }_{-} \\
\text {VIOLENCIA }\end{array}$ & $\begin{array}{l}\mathrm{C}_{-} \mathrm{PRO} \\
\text { SOCIALES }\end{array}$ & $\frac{\mathrm{C}_{-}}{\text {AGRESIVAS }}$ & CIBERACOSO \\
\hline \multirow[t]{3}{*}{ AUTOACEPTACIÓN } & -0.24 & -0.16 & 0.32 & -0.10 & -0.07 \\
\hline & 0.00 & 0.02 & 0.00 & 0.14 & 0.29 \\
\hline & 217.00 & 219.00 & 219.00 & 217.00 & 219.00 \\
\hline \multirow[t]{3}{*}{ RE_POST } & 0.18 & 0.14 & -0.26 & -0.02 & 0.09 \\
\hline & 0.01 & 0.04 & 0.00 & 0.76 & 0.20 \\
\hline & 217.00 & 219.00 & 219.00 & 217.00 & 219.00 \\
\hline \multirow[t]{3}{*}{ AUTONOMÍA } & 0.09 & 0.12 & -0.20 & -0.06 & 0.05 \\
\hline & 0.17 & 0.07 & 0.00 & 0.41 & 0.50 \\
\hline & 217.00 & 219.00 & 219.00 & 217.00 & 219.00 \\
\hline \multirow[t]{3}{*}{ DOM_ENTORNO } & -0.28 & -0.14 & 0.22 & -0.04 & -0.13 \\
\hline & 0.00 & 0.04 & 0.00 & 0.54 & 0.06 \\
\hline & 217.00 & 219.00 & 219.00 & 217.00 & 219.00 \\
\hline \multirow[t]{3}{*}{ CRECI_PERSONAL } & -0.25 & -0.12 & 0.19 & -0.03 & -0.04 \\
\hline & 0.00 & 0.08 & 0.01 & 0.64 & 0.52 \\
\hline & 217.00 & 219.00 & 219.00 & 217.00 & 219.00 \\
\hline \multirow[t]{3}{*}{ PROPÓSITO_VIDA } & -0.22 & -0.11 & 0.21 & -0.06 & -0.04 \\
\hline & 0.00 & 0.12 & 0.00 & 0.41 & 0.54 \\
\hline & 217.00 & 219.00 & 219.00 & 217.00 & 219.00 \\
\hline
\end{tabular}


Respecto al grupo etario conformado por jóvenes (tabla 10), la subescala de influencia externa se correlaciona de manera positiva con el área de relaciones positivas, lo cual indicaría que ante una mayor influencia que los jóvenes tienen sobre las conductas agresivas de sus pares mayor es calidad de las relaciones con los mismos; asimismo, esta subescala se correlaciona negativamente con las áreas de dominio del entorno, crecimiento personal y propósito de vida, por lo que se puede inferir que mientras mayor sea la influencia que los jóvenes tienen sobre las conductas agresivas de sus pares menor es el manejo de las oportunidades y exigencias del medio, el aprovechamiento de sus potencialidades y la claridad para definir sus metas.

Por otro lado, existe una correlación positiva entre la subescala conductas pro sociales y las áreas de autoaceptación, dominio del entorno, crecimiento personal y propósito de vida, a partir de lo cual se puede inferir que a mayor ratio de conductas solidarias con sus pares mayor es la aceptación de los diversos aspectos de su personalidad, el manejo de las exigencias y oportunidades del ambiente, el aprovechamiento de sus habilidades y la claridad en la definición de sus metas. Asimismo, dicha sub escala se correlaciona negativamente con el área de relaciones positivas, por lo cual puede inferirse que a mayor índice de conductas solidarias menor es la calidad de las relaciones que se sostienen con los pares.

Tabla 10. Correlación entre las subescalas de la agresión entre pares y las áreas del bienestar psicológico en función a la edad. Jóvenes.

\begin{tabular}{|c|c|c|c|c|c|}
\hline \multicolumn{6}{|c|}{ Correlaciones según edad en jóvenes } \\
\hline & $\begin{array}{c}\text { INF } \\
\text { EXTERNA }\end{array}$ & $\begin{array}{c}\mathrm{ACT}_{-} \\
\text {VIOLENCIA }\end{array}$ & $\begin{array}{l}\mathrm{C}_{-} \text {PRO_-} \\
\text { SOCIALES }\end{array}$ & $\frac{\mathrm{C}_{-}}{\text {AGRESIVAS }}$ & CIBERACOSO \\
\hline \multirow[t]{3}{*}{ AUTOACEPTACIÓN } & -0.192 & -0.176 & 0.308 & -0.084 & -0.094 \\
\hline & 0.000 & 0.000 & 0.000 & 0.012 & 0.005 \\
\hline & 893.000 & 895.000 & 895.000 & 894.000 & 895.000 \\
\hline \multirow[t]{3}{*}{ RE_POST } & 0.256 & 0.194 & -0.337 & 0.134 & 0.155 \\
\hline & 0.000 & 0.000 & 0.000 & 0.000 & 0.000 \\
\hline & 895.000 & 897.000 & 897.000 & 896.000 & 897.000 \\
\hline \multirow[t]{3}{*}{ AUTONOMÍA } & 0.092 & 0.157 & -0.154 & 0.038 & 0.093 \\
\hline & 0.006 & 0.000 & 0.000 & 0.253 & 0.005 \\
\hline & 894.000 & 896.000 & 896.000 & 895.000 & 896.000 \\
\hline \multirow[t]{3}{*}{ DOM_ENTORNO } & -0.201 & -0.176 & 0.245 & -0.072 & -0.102 \\
\hline & 0.000 & 0.000 & 0.000 & 0.031 & 0.002 \\
\hline & 894.000 & 896.000 & 896.000 & 896.000 & 896.000 \\
\hline \multirow[t]{2}{*}{ CRECI_PERSONAL } & -0.216 & -0.170 & 0.361 & -0.083 & -0.125 \\
\hline & 0.000 & 0.000 & 0.000 & 0.013 & 0.000 \\
\hline
\end{tabular}


Carlos Velásquez C., William Montgomery U., Alejandro Dioses C., Ricardo Pomalaya V., José Chávez Z.

$\begin{array}{cccccc} & 895.000 & 897.000 & 897.000 & 896.000 & 897.000 \\ \text { PROPÓSITO_VIDA } & -0.211 & -0.126 & 0.313 & -0.110 & -0.120 \\ & 0.000 & 0.000 & 0.000 & 0.001 & 0.000 \\ & 892.000 & 894.000 & 894.000 & 893.000 & 894.000\end{array}$

En relación al grupo etario conformado por adultos (tabla 11), existe una correlación positiva entre la subescala de influencia externa y las áreas de relaciones positivas y autonomía, lo cual indicaría que ante una mayor influencia que los jóvenes tienen sobre las conductas agresivas de sus pares, mayor es la calidad de las relaciones que tienen con su compañeros así como la capacidad para tomar decisiones de manera independiente. Asimismo, esta subescala se correlaciona negativamente con el área de dominio del entorno, por lo que se puede inferir que mientras mayor sea la influencia que los jóvenes tienen sobre las conductas agresivas de sus pares, menor es el manejo de las oportunidades y exigencias del medio. La subescala, actitud hacia la violencia, se correlaciona de manera positiva con autonomía, lo que indicaría que a mayor actitud frente a conductas agresivas es mayor la capacidad para tomar decisiones. Igualmente, dicha subescala se correlaciona de manera negativa con el área dominio del entorno, con lo cual podemos afirmar que a mayor actitud frente a conductas agresivas es menor el manejo de las exigencias y las oportunidades del ambiente. Por otro lado, existe una correlación positiva entre la subescala conductas prosociales y las áreas de autoaceptación, dominio del entorno, crecimiento personal y propósito de vida, a partir de lo cual se puede colegir que a mayor ratio de conductas solidarias con sus pares mayor es la aceptación de los diversos aspectos de su personalidad, el manejo de las exigencias y oportunidades del ambiente, el aprovechamiento de sus habilidades y la claridad en la definición de sus metas. Asimismo, dicha subescala se correlaciona negativamente con el área de relaciones positivas y autonomía, por lo cual podemos inferir que a mayor grado de conductas solidarias menor es la calidad de las relaciones que se sostiene con los pares así como la capacidad para tomar decisiones de manera independiente.

La subescala de conductas agresivas se correlaciona de manera positiva con la de relaciones positivas, con lo cual se puede inferir que a mayor participación en conductas agresivas es mayor la calidad de las relaciones que sostiene con sus pares. Asimismo, dicha subescala se correlaciona de manera negativa con el área dominio del entorno, con lo cual podemos inferir que a mayor actitud frente a las conductas agresivas es menor el manejo de las exigencias y las oportunidades del ambiente. La subescala ciberacoso se correlaciona de manera positiva con relaciones positivas, con lo cual se puede inferir que a mayor participación en conductas agresivas por medios virtuales es mayor la calidad de las relaciones que sostiene con sus pares; asimismo, esa subescala se correlaciona de manera negativa con el área dominio del entorno, con lo cual se deduce que a mayor participación de conductas agresivas por medios virtuales, es menor el manejo de las exigencias y las oportunidades del ambiente. 
Tabla 11. Correlación entre las subescalas de la agresión entre pares y las áreas del bienestar psicológico en función a la edad. Adultos.

\begin{tabular}{cccccc}
\hline \multicolumn{5}{c}{ Correlaciones según edad en adultos } & \\
\hline & INF_ & ACT_ & C_PRO_ & C__ & CIBERACOSO \\
& EXTERNA & VIOLENCIA & SOCIALES & AGRESIVAS & \\
\hline AUTOACEPTACIÓN & -0.186 & 0.039 & 0.424 & -0.109 & -0.078 \\
& 0.187 & 0.782 & 0.002 & 0.442 & 0.582 \\
& 52.000 & 52.000 & 52.000 & 52.000 & 52.000 \\
RE_POST & 0.374 & 0.143 & -0.319 & 0.322 & 0.230 \\
& 0.006 & 0.312 & 0.021 & 0.020 & 0.100 \\
AUTONOMÍA & 52.000 & 52.000 & 52.000 & 52.000 & 52.000 \\
& 0.286 & 0.348 & -0.442 & 0.134 & 0.182 \\
& 0.040 & 0.012 & 0.001 & 0.343 & 0.197 \\
DOM_ENTORNO & 52.000 & 52.000 & 52.000 & 52.000 & 52.000 \\
& -0.328 & -0.234 & 0.331 & -0.228 & -0.237 \\
& 0.018 & 0.095 & 0.017 & 0.104 & 0.091 \\
CRECI_PERSONAL & 52.000 & 52.000 & 52.000 & 52.000 & 52.000 \\
& -0.062 & 0.004 & 0.242 & -0.070 & 0.042 \\
& 0.662 & 0.980 & 0.084 & 0.621 & 0.768 \\
& 52.000 & 52.000 & 52.000 & 52.000 & 52.000 \\
& -0.098 & 0.124 & 0.258 & -0.099 & -0.125 \\
& 0.489 & 0.383 & 0.065 & 0.485 & 0.377 \\
& 52.000 & 52.000 & 52.000 & 52.000 & 52.000 \\
\hline
\end{tabular}

En el género masculino (tabla 12), existe una correlación positiva entre la sub escala influencia externa y el área de relaciones positivas, lo que señalaría que ante una mayor influencia que se tiene sobre las conductas agresivas en los pares mayor es la calidad de las relaciones con los demás. La subescala de conductas pro sociales se correlaciona positivamente con las áreas de autoaceptación, crecimiento personal y propósito de vida, a partir de lo cual se puede inferir que a mayores conductas solidarias con sus pares mayor es la aceptación de sí mismo, el aprovechamiento de sus potencialidades y la claridad en la definición de sus metas. Igualmente, la subescala de conductas pro sociales se correlaciona negativamente con el área de relaciones positivas, lo que indicaría que a medida de que las conductas solidarias con sus pares aumentan, la calidad de las relaciones disminuye.

Tabla 12. Correlación entre las subescalas de la agresión entre pares y las áreas del bienestar psicológico en función del género. Masculino.

\begin{tabular}{cccccc}
\hline \multicolumn{6}{c}{ Correlaciones según género masculino } \\
\hline & $\begin{array}{c}\text { INF_ } \\
\text { EXTERNA }\end{array}$ & $\begin{array}{c}\text { ACT_ } \\
\text { VIOLENCIA }\end{array}$ & $\begin{array}{c}\text { C_PRO_ } \\
\text { SOCIALES }\end{array}$ & $\begin{array}{c}\text { C_- } \\
\text { AGRESIVAS }\end{array}$ & CIBERACOSO \\
\hline AUTOACEPTACIÓN & -0.196 & -0.185 & 0.327 & -0.112 & -0.132 \\
& 0.000 & 0.000 & 0.000 & 0.004 & 0.001 \\
& 671.000 & 673.000 & 673.000 & 672.000 & 673.000
\end{tabular}




\begin{tabular}{cccccc} 
RE_POST & 0.200 & 0.105 & -0.276 & 0.109 & 0.116 \\
& 0.000 & 0.006 & 0.000 & 0.005 & 0.003 \\
AUTONOMÍA & 673.000 & 675.000 & 675.000 & 674.000 & 675.000 \\
& 0.091 & 0.158 & -0.138 & 0.045 & 0.148 \\
& 0.018 & 0.000 & 0.000 & 0.241 & 0.000 \\
DOM_ENTORNO & 673.000 & 675.000 & 675.000 & 674.000 & 675.000 \\
& -0.190 & -0.174 & 0.254 & -0.076 & -0.127 \\
CRECI_PERSONAL & 0.000 & 0.000 & 0.000 & 0.049 & 0.001 \\
& 673.000 & 675.000 & 675.000 & 674.000 & 675.000 \\
& -0.159 & -0.093 & 0.348 & -0.051 & -0.078 \\
PROPÓSITO_VIDA & 0.000 & 0.016 & 0.000 & 0.189 & 0.042 \\
& 673.000 & 675.000 & 675.000 & 674.000 & 675.000 \\
& -0.194 & -0.101 & 0.302 & -0.117 & -0.122 \\
& 0.000 & 0.009 & 0.000 & 0.002 & 0.001 \\
& 672.000 & 674.000 & 674.000 & 673.000 & 674.000 \\
\hline
\end{tabular}

En el género femenino (tabla 13), la subescala de influencia externa correlaciona positivamente con el área de relaciones positivas, lo que señalaría que a mayor influencia que se tiene sobre la conducta agresiva de los pares mayor es la calidad de las relaciones positivas; asimismo, dicha subescala correlaciona negativamente con las áreas de autoaceptación, dominio del entorno, crecimiento personal y propósito de vida, lo cual indicaría que a mayor influencia que tiene el género femenino sobre las conductas de agresión entre pares, menor es la aceptación de sí mismas con actitud positiva, el buen manejo de las exigencias y oportunidades del ambiente, el aprovechamiento de sus habilidades y la claridad para definir sus metas y objetivos. Por otro lado, la subescala de actitud hacia la violencia se correlaciona positivamente con las áreas de autoaceptación y autonomía, lo que señalaría que mientras mayor es la actitud personal hacia la violencia y los modos alternativos de resolver conflictos mayor es la calidad de las relaciones con los demás y la capacidad para tomar sus propias decisiones; asimismo, dicha subescala correlaciona negativamente con el área de crecimiento personal, lo que indicaría que a mayor actitud hacia la violencia y los modos alternativos de solucionar los conflictos menor es el aprovechamiento de sus habilidades. Finalmente, la subescala de conductas pro sociales correlaciona positivamente con las áreas de autoaceptación, dominio del entorno, crecimiento personal y propósito en la vida, con lo cual podemos inferir que ante una mayor conducta solidaria mayor es la aceptación de diversos aspectos de su personalidad con actitud positiva, el manejo de las exigencias y oportunidad del ambiente, al aprovechamiento de sus potencialidades y la claridad para definir sus objetivos. Asimismo, dicha subescala correlaciona negativamente con las áreas de relaciones positivas y autonomía, lo que indicaría que mientras mayor sean las conductas solidarias con los demás menor será la calidad de relaciones con los pares y la capacidad para tomar decisiones, manteniendo cierta dependencia personal. 
Tabla 13. Correlación entre las subescalas de la agresión entre pares y las áreas del bienestar psicológico en función del género. Mujeres.

\begin{tabular}{|c|c|c|c|c|c|}
\hline \multicolumn{6}{|c|}{ Correlaciones según género femenino } \\
\hline & $\begin{array}{c}\text { INF } \\
\text { EXTERNA }\end{array}$ & $\begin{array}{c}\text { ACT }_{-} \\
\text {VIOLENCIA }\end{array}$ & $\begin{array}{l}\mathrm{C}_{-} \mathrm{PRO} \\
\text { SOCIALES }\end{array}$ & $\begin{array}{c}\mathrm{C} \\
\text { AGRESIVAS }\end{array}$ & CIBERACOSO \\
\hline \multirow[t]{3}{*}{ AUTOACEPTACIÓN } & -0.245 & -0.161 & 0.301 & -0.076 & -0.042 \\
\hline & 0.000 & 0.000 & 0.000 & 0.094 & 0.356 \\
\hline & 491.000 & 493.000 & 493.000 & 491.000 & 493.000 \\
\hline \multirow[t]{3}{*}{ RE_POST } & 0.313 & 0.283 & -0.370 & 0.115 & 0.174 \\
\hline & 0.000 & 0.000 & 0.000 & 0.011 & 0.000 \\
\hline & 491.000 & 493.000 & 493.000 & 491.000 & 493.000 \\
\hline \multirow[t]{3}{*}{ AUTONOMÍA } & 0.176 & 0.226 & -0.245 & 0.049 & 0.035 \\
\hline & 0.000 & 0.000 & 0.000 & 0.282 & 0.435 \\
\hline & 490.000 & 492.000 & 492.000 & 490.000 & 492.000 \\
\hline \multirow[t]{3}{*}{ DOM_ENTORNO } & -0.294 & -0.174 & 0.239 & -0.080 & -0.091 \\
\hline & 0.000 & 0.000 & 0.000 & 0.075 & 0.044 \\
\hline & 490.000 & 492.000 & 492.000 & 491.000 & 492.000 \\
\hline \multirow[t]{3}{*}{ CRECI_PERSONAL } & -0.291 & -0.213 & 0.281 & -0.098 & -0.132 \\
\hline & 0.000 & 0.000 & 0.000 & 0.030 & 0.003 \\
\hline & 491.000 & 493.000 & 493.000 & 491.000 & 493.000 \\
\hline \multirow[t]{3}{*}{ PROPÓSITO_VIDA } & -0.207 & -0.083 & 0.259 & -0.049 & -0.051 \\
\hline & 0.000 & 0.065 & 0.000 & 0.278 & 0.255 \\
\hline & 489.000 & 491.000 & 491.000 & 489.000 & 491.000 \\
\hline
\end{tabular}

En las Ciencias de la Salud (tabla 14), la sub escala de influencia externa correlaciona positivamente con el área de relaciones positivas, lo que indicaría que mientras mayor sea la influencia sobre las conductas agresivas de los demás, mayor es la calidad de las relaciones con los demás. La sub escala de conductas prosociales correlaciona positivamente con las áreas de autoaceptación, crecimiento personal y propósito de vida, lo que señalaría que a mayor conductas solidarias mayor será la aceptación de diversos aspectos de su personalidad con actitud positiva, el aprovechamiento de sus potencialidades y la claridad para definir sus metas. Asimismo, dicha subescala correlaciona negativamente con el área de relaciones positivas, lo que indicaría que a mayor grado de conductas solidarias con sus pares menor es la calidad de las relaciones con los demás. Por último, la subescala ciberacoso correlaciona negativamente con el área de autonomía, con lo que se puede afirmar que a mayor utilización de medios virtuales para expresar violencia menor es la capacidad para tomar decisiones de manera independiente. 
Tabla 14. Correlación entre las sub escalas de la agresión entre pares y las áreas del bienestar psicológico en función a las áreas profesionales: Ciencias de la Salud.

\begin{tabular}{|c|c|c|c|c|c|}
\hline \multicolumn{6}{|c|}{ Correlaciones según áreas en ciencias de la salud } \\
\hline & $\begin{array}{c}\text { INF } \\
\text { EXTERNA }\end{array}$ & $\begin{array}{c}\text { ACT }_{-} \\
\text {VIOLENCIA }\end{array}$ & $\begin{array}{c}\mathrm{C}_{-} \text {PRO_- } \\
\text { SOCIALES }\end{array}$ & $\frac{\mathrm{C}_{-}}{\text {AGRESIVAS }}$ & CIBERACOSO \\
\hline \multirow[t]{3}{*}{ AUTOACEPTACIÓN } & -0.037 & 0.085 & 0.358 & -0.001 & 0.039 \\
\hline & 0.714 & 0.395 & 0.000 & 0.992 & 0.701 \\
\hline & 101.000 & 101.000 & 101.000 & 99.000 & 101.000 \\
\hline \multirow[t]{3}{*}{ RE_POST } & 0.284 & 0.177 & -0.475 & 0.199 & 0.174 \\
\hline & 0.004 & 0.076 & 0.000 & 0.048 & 0.082 \\
\hline & 101.000 & 101.000 & 101.000 & 99.000 & 101.000 \\
\hline \multirow[t]{3}{*}{ AUTONOMÍA } & -0.050 & -0.006 & -0.105 & -0.174 & -0.301 \\
\hline & 0.619 & 0.951 & 0.297 & 0.085 & 0.002 \\
\hline & 101.000 & 101.000 & 101.000 & 99.000 & 101.000 \\
\hline \multirow[t]{3}{*}{ DOM_ENTORNO } & -0.162 & -0.045 & 0.141 & -0.177 & -0.096 \\
\hline & 0.107 & 0.654 & 0.161 & 0.080 & 0.343 \\
\hline & 100.000 & 100.000 & 100.000 & 99.000 & 100.000 \\
\hline \multirow[t]{3}{*}{ CRECI_PERSONAL } & -0.168 & -0.075 & 0.340 & -0.153 & -0.134 \\
\hline & 0.092 & 0.457 & 0.001 & 0.130 & 0.183 \\
\hline & 101.000 & 101.000 & 101.000 & 99.000 & 101.000 \\
\hline \multirow[t]{3}{*}{ PROPÓSITO_VIDA } & 0.035 & 0.006 & 0.330 & -0.071 & -0.058 \\
\hline & 0.732 & 0.952 & 0.001 & 0.487 & 0.565 \\
\hline & 100.000 & 100.000 & 100.000 & 98.000 & 100.000 \\
\hline
\end{tabular}

En el área de Humanidades (tabla 15), la subescala conductas pro sociales correlaciona positivamente con el área de autoaceptación, dominio del entorno, crecimiento personal y propósito de vida, lo que indicaría que mientras mayor sea la conducta solidaria con los demás mayor es la aceptación de sí mismos con actitud positiva, el manejo de exigencias y oportunidades del ambiente, el aprovechamiento de sus potencialidades y la claridad en la definición de objetivos. Asimismo, dicha subescala correlaciona negativamente con el área de relaciones positivas, lo cual indicaría que mientras mayores sean las conductas solidarias para con sus pares menor es la calidad de la relación. La subescala de ciberacoso se correlaciona positivamente con el área de autonomía, lo cual señalaría que a mayor acoso virtual mayor será la capacidad para tomar decisiones de manera independiente. Asimismo, dicha subescala se correlaciona negativamente con las áreas autoaceptación y propósito en la vida, lo que referiría que mientras mayor sea el acoso virtual, menor será la aceptación de sí mismo y la claridad en la definición de metas. 
Tabla 15. Correlación entre las sub escalas de la agresión entre pares y las áreas del bienestar psicológico en función a las áreas profesionales: Humanidades.

\begin{tabular}{cccccc}
\hline \multicolumn{5}{c}{ Correlaciones según áreas en humanidades } & \\
\hline & INF__ & ACT_ & C_PRO_ & C__ & CIBERACOSO \\
& EXTERNA & VIOLENCIA & SOCIALES & AGRESIVAS & \\
\hline AUTOACEPTACIÓN & -0.156 & -0.103 & 0.347 & -0.113 & -0.201 \\
& 0.016 & 0.110 & 0.000 & 0.081 & 0.002 \\
& 237.000 & 241.000 & 241.000 & 241.000 & 241.000 \\
RE_POST & 0.127 & 0.010 & -0.205 & 0.087 & 0.081 \\
& 0.051 & 0.875 & 0.001 & 0.179 & 0.210 \\
AUTONOMÍA & 238.000 & 242.000 & 242.000 & 242.000 & 242.000 \\
& 0.081 & 0.074 & -0.080 & -0.013 & 0.277 \\
& 0.212 & 0.249 & 0.215 & 0.836 & 0.000 \\
DOM_ENTORNO & 238.000 & 242.000 & 242.000 & 242.000 & 242.000 \\
& -0.195 & -0.048 & 0.204 & -0.017 & -0.193 \\
& 0.003 & 0.461 & 0.001 & 0.796 & 0.003 \\
& 238.000 & 242.000 & 242.000 & 242.000 & 242.000 \\
CRECI_PERSONAL & -0.132 & -0.031 & 0.262 & -0.105 & -0.171 \\
& 0.042 & 0.636 & 0.000 & 0.104 & 0.008 \\
& 238.000 & 242.000 & 242.000 & 242.000 & 242.000 \\
& -0.197 & -0.109 & 0.258 & -0.128 & -0.225 \\
& 0.002 & 0.090 & 0.000 & 0.048 & 0.000 \\
& 237.000 & 241.000 & 241.000 & 241.000 & 241.000 \\
\hline
\end{tabular}

En el área de Ciencias sociales (tabla 16), la subescala de influencia externa se correlaciona positivamente con las áreas de relaciones positivas y autonomía, lo cual señalaría que a mayor influencia que se tenga sobre las conductas agresivas de los demás, mayor será la calidad de las relaciones con los pares y la capacidad para tomar decisiones propias. Igualmente, dicha sub escala se correlaciona negativamente con las áreas de dominio del entorno, crecimiento personal y propósito de vida, lo que referiría que mientras mayor sea la influencia sobre las conductas agresivas de sus compañeros, menor será la capacidad para manejar las exigencias y oportunidades del entorno así como el aprovechamiento de las potencialidades y la definición de metas. La subescala de actitud hacia la violencia se correlaciona positivamente con las áreas de relaciones positivas y autonomía, indicando que mientras mayor sea la conducta agresiva para con los demás mayor será la calidad de las relaciones con los pares y la capacidad para tomar decisiones propias. Así también, dicha subescala se correlaciona negativamente con las áreas de autoaceptación, dominio del entorno y crecimiento personal, lo que referiría que mientras mayor sea el comportamiento agresivo con sus pares menor será la aceptación de sí mismos con una actitud positiva, la capacidad para manejar las exigencias y oportunidades del entorno así como el aprovechamiento de las potencialidades. La subescala de conductas prosociales se correlaciona positivamente con las áreas de 
autoaceptación, dominio del entorno, crecimiento personal y propósito en la vida, lo cual señalaría que mientras mayor sea la conducta solidaria hacia los demás mayor será la aceptación de sí mismos, el manejo de las exigencias y oportunidades del medio, el aprovechamiento de sus habilidades y la claridad de los objetivos para su vida; de otro lado, dicha subescala se correlaciona negativamente con las áreas de relaciones positivas y autonomía, a partir de esto se puede inferir que mientras mayor es la conducta solidaria es menor la calidad de las relaciones con los pares, así como la capacidad para tomar decisiones de manera independiente al margen de los demás.

Tabla 16. Correlación entre las sub escalas de la Agresión entre pares y las áreas del bienestar psicológico en función a las áreas profesionales: Ciencias Sociales.

\begin{tabular}{cccccc}
\hline \multicolumn{5}{c}{ Correlaciones según áreas en ciencias sociales } & \\
\hline & INF_- & ACT_ & C_PRO_ & C__ & CIBERACOSO \\
& EXTERNA & VIOLENCIA & SOCIALES & AGRESIVAS & \\
\hline AUTOACEPTACIÓN & -0.220 & -0.159 & 0.395 & 0.001 & -0.043 \\
& 0.002 & 0.024 & 0.000 & 0.994 & 0.542 \\
& 201.000 & 201.000 & 201.000 & 200.000 & 201.000 \\
RE_POST & 0.345 & 0.281 & -0.417 & 0.127 & 0.232 \\
& 0.000 & 0.000 & 0.000 & 0.074 & 0.001 \\
AUTONOMÍA & 201.000 & 201.000 & 201.000 & 200.000 & 201.000 \\
& 0.169 & 0.177 & -0.188 & 0.090 & 0.025 \\
& 0.017 & 0.012 & 0.008 & 0.205 & 0.724 \\
DOM_ENTORNO & 200.000 & 200.000 & 200.000 & 199.000 & 200.000 \\
& -0.338 & -0.188 & 0.420 & -0.075 & -0.092 \\
& 0.000 & 0.008 & 0.000 & 0.293 & 0.192 \\
& 201.000 & 201.000 & 201.000 & 200.000 & 201.000 \\
CRECI_PERSONAL & -0.273 & -0.169 & 0.476 & -0.078 & -0.121 \\
& 0.000 & 0.016 & 0.000 & 0.272 & 0.088 \\
& 201.000 & 201.000 & 201.000 & 200.000 & 201.000 \\
& -0.305 & -0.212 & 0.326 & -0.156 & -0.120 \\
& 0.000 & 0.003 & 0.000 & 0.028 & 0.091 \\
\hline
\end{tabular}

En las Ciencias Básicas (tabla 17), la subescala de influencia externa se correlaciona positivamente con las áreas de relaciones positivas y autonomía, lo cual señalaría que a mayor influencia que se tenga sobre las conductas agresivas de los demás, mayor será la calidad de las relaciones con los pares y la capacidad para tomar decisiones propias; asimismo, dicha subescala se correlaciona negativamente con las áreas de dominio del entorno y crecimiento personal, lo que referiría que mientras mayor sea la influencia sobre las conductas agresivas de sus compañeros menor será la capacidad para manejar las exigencias y oportunidades del entorno así como el aprovechamiento de las potencialidades. La subescala de actitud hacia la violencia se correlaciona positivamente con las áreas de relaciones positivas y 
autonomía, lo cual indicaría que mientras mayor sea la conducta agresiva para con los demás mayor será la calidad de las relaciones con los pares y la capacidad para tomar decisiones propias; así también, dicha subescala se correlaciona negativamente con las áreas de autoaceptación, dominio del entorno y crecimiento personal, lo que deduce que mientras mayor sea el comportamiento agresivo con sus pares menor será la aceptación de sí mismos con una actitud positiva, la capacidad para manejar las exigencias y oportunidades del entorno así como el aprovechamiento de las potencialidades. La subescala de conductas pro sociales se correlaciona positivamente con las áreas de autoaceptación, dominio del entorno, crecimiento personal y propósito en la vida, lo cual señalaría que mientras mayor sea la conducta solidaria hacia los demás mayor será la aceptación de sí mismos, el manejo de las exigencias y oportunidades del medio, el aprovechamiento de sus habilidades y la claridad de los objetivos para su vida; de otro lado, dicha subescala se correlaciona negativamente con las áreas de relaciones positivas y autonomía, a partir de esto se puede inferir que mientras mayor es la conducta solidaria es menor la calidad de las relaciones con los pares, así como la capacidad para tomar decisiones de manera independiente al margen de los demás.

Tabla 17. Correlación entre las subescalas de la agresión entre pares y las áreas del bienestar psicológico en función a las áreas profesionales: Ciencias Básicas.

\begin{tabular}{|c|c|c|c|c|c|}
\hline \multicolumn{6}{|c|}{ Correlaciones según áreas en ciencias básicas } \\
\hline & $\begin{array}{c}\text { INF- } \\
\text { EXTERNA }\end{array}$ & $\begin{array}{c}\text { ACT } \\
\text { VIOLENCIA }\end{array}$ & $\begin{array}{l}\text { C_PRO_- } \\
\text { SOCIALES }\end{array}$ & $\begin{array}{c}\mathrm{C} \\
\text { AGRESIVAS }\end{array}$ & CIBERACOSO \\
\hline \multirow[t]{3}{*}{ AUTOACEPTACIÓN } & -0.196 & -0.231 & 0.276 & -0.145 & 0.013 \\
\hline & 0.016 & 0.005 & 0.001 & 0.077 & 0.875 \\
\hline & 150.000 & 150.000 & 150.000 & 150.000 & 150.000 \\
\hline \multirow[t]{3}{*}{ RE_POST } & 0.316 & 0.247 & -0.405 & 0.197 & 0.189 \\
\hline & 0.000 & 0.002 & 0.000 & 0.016 & 0.020 \\
\hline & 150.000 & 150.000 & 150.000 & 150.000 & 150.000 \\
\hline \multirow[t]{3}{*}{ AUTONOMÍA } & 0.262 & 0.262 & -0.265 & 0.092 & 0.116 \\
\hline & 0.001 & 0.001 & 0.001 & 0.265 & 0.159 \\
\hline & 150.000 & 150.000 & 150.000 & 150.000 & 150.000 \\
\hline \multirow[t]{3}{*}{ DOM_ENTORNO } & -0.258 & -0.290 & 0.267 & -0.088 & 0.034 \\
\hline & 0.001 & 0.000 & 0.001 & 0.286 & 0.679 \\
\hline & 150.000 & 150.000 & 150.000 & 150.000 & 150.000 \\
\hline \multirow[t]{3}{*}{ CRECI_PERSONAL } & -0.225 & -0.262 & 0.383 & 0.017 & 0.007 \\
\hline & 0.006 & 0.001 & 0.000 & 0.838 & 0.934 \\
\hline & 150.000 & 150.000 & 150.000 & 150.000 & 150.000 \\
\hline \multirow[t]{3}{*}{ PROPÓSITO_VIDA } & -0.222 & -0.177 & 0.305 & -0.099 & 0.038 \\
\hline & 0.006 & 0.030 & 0.000 & 0.230 & 0.648 \\
\hline & 150.000 & 150.000 & 150.000 & 150.000 & 150.000 \\
\hline
\end{tabular}


En el área de Ingenierías (tabla 18), la subescala de influencia externa se correlaciona negativamente con el área de autoaceptación, lo cual señalaría que a mayor influencia que se tenga sobre las conductas agresivas de los pares, menor será la aceptación de los diversos aspectos de su personalidad de manera positiva. La sub escala de conductas pro sociales se correlaciona positivamente con las áreas de autoaceptación, dominio del entorno, crecimiento personal y propósito en la vida, a partir de lo cual se puede inferir que mientras más solidario se es con los demás mayor será la aceptación de sí mismos, el manejo de las exigencias y oportunidades del medio, el aprovechamiento de sus habilidades y la claridad de los objetivos para su vida. Asimismo, dicha subescala se correlaciona negativamente con el área de relaciones positivas, de esta manera se puede afirmar que mientras mayor sea la conducta solidaria es menor la calidad de las relaciones que se mantiene con los compañeros. La subescala de conductas agresivas se correlaciona negativamente con el área de autoaceptación, por lo que se puede colegir que mientras mayor agresivo se es con los pares menor aceptación de los aspectos de su personalidad poseerá.

Tabla 18. Correlación entre las sub escalas de la agresión entre pares y las áreas del bienestar psicológico en función a las áreas profesionales: Ingeniería.

\begin{tabular}{|c|c|c|c|c|c|}
\hline \multicolumn{6}{|c|}{ Correlaciones según áreas en ingenierías } \\
\hline & $\begin{array}{c}\text { INF- } \\
\text { EXTERNA }\end{array}$ & $\begin{array}{c}\mathrm{ACT}_{-} \\
\text {VIOLENCIA }\end{array}$ & $\begin{array}{c}\text { C_PRO_- }_{-} \\
\text {SOCIALES }\end{array}$ & $\begin{array}{c}\mathrm{C}_{-} \\
\text {AGRESIVAS }\end{array}$ & CIBERACOSO \\
\hline \multirow[t]{3}{*}{ AUTOACEPTACIÓN } & -0.202 & -0.174 & 0.272 & -0.204 & -0.145 \\
\hline & 0.001 & 0.004 & 0.000 & 0.001 & 0.016 \\
\hline & 274.000 & 274.000 & 274.000 & 274.000 & 274.000 \\
\hline \multirow[t]{3}{*}{ RE_POST } & 0.142 & 0.131 & -0.320 & 0.112 & 0.099 \\
\hline & 0.019 & 0.031 & 0.000 & 0.065 & 0.102 \\
\hline & 274.000 & 274.000 & 274.000 & 274.000 & 274.000 \\
\hline \multirow[t]{3}{*}{ AUTONOMÍA } & -0.011 & 0.130 & -0.146 & 0.091 & 0.148 \\
\hline & 0.852 & 0.031 & 0.015 & 0.133 & 0.014 \\
\hline & 274.000 & 274.000 & 274.000 & 274.000 & 274.000 \\
\hline \multirow[t]{3}{*}{ DOM_ENTORNO } & -0.074 & -0.082 & 0.204 & -0.116 & -0.149 \\
\hline & 0.222 & 0.177 & 0.001 & 0.055 & 0.013 \\
\hline & 274.000 & 274.000 & 274.000 & 274.000 & 274.000 \\
\hline \multirow[t]{3}{*}{ CRECI_PERSONAL } & -0.132 & -0.154 & 0.310 & -0.153 & -0.117 \\
\hline & 0.029 & 0.011 & 0.000 & 0.011 & 0.052 \\
\hline & 274.000 & 274.000 & 274.000 & 274.000 & 274.000 \\
\hline \multirow[t]{3}{*}{ PROPÓSITO_VIDA } & -0.152 & -0.143 & 0.308 & -0.111 & -0.075 \\
\hline & 0.012 & 0.018 & 0.000 & 0.068 & 0.217 \\
\hline & 274.000 & 274.000 & 274.000 & 274.000 & 274.000 \\
\hline
\end{tabular}


En el área económico-empresarial (tabla 19), la subescala de influencia externa se correlaciona positivamente con el área de relaciones positivas, lo cual indicaría que a mayor influencia que se tenga sobre las conductas agresivas de los demás mayor será la calidad de las relaciones con los demás; asimismo, dicha subescala se correlaciona negativamente con las áreas de autoaceptación, dominio del entorno, crecimiento personal y propósito de la vida, lo que referiría que mientras mayor sea la influencia sobre las conductas agresivas de sus compañeros menor será la aceptación de sí mismo de manera positiva, la capacidad para manejar las exigencias y oportunidades del entorno, el aprovechamiento de las potencialidades y la claridad para definir sus metas. La sub escala de actitud hacia la violencia se correlaciona positivamente con las áreas de relaciones positivas y autonomía, con lo cual se puede afirmar que mientras mayor sea la conducta agresiva para con los demás, mayor será la calidad de las relaciones con los pares y la capacidad para tomar decisiones propias. De otro lado, dicha sub escala se correlaciona negativamente con las áreas de autoaceptación y dominio del entorno, lo que referiría que mientras mayor sea el comportamiento agresivo con sus pares menor será la aceptación de sí mismos con una actitud positiva y la capacidad para manejar las exigencias y oportunidades del entorno. La subescala de conductas pro sociales se correlaciona positivamente con las áreas de autoaceptación, crecimiento personal y propósito de vida, lo cual señalaría que mientras mayor sea la conducta solidaria hacia los demás mayor será la aceptación de sí mismos, el aprovechamiento de sus habilidades y la claridad para definir sus metas.

Tabla 19. Correlación entre las sub escalas de la agresión entre pares y las áreas del bienestar psicológico en función a las áreas profesionales: Económico-Empresariales.

\begin{tabular}{cccccc}
\hline \multicolumn{5}{c}{ Correlaciones según áreas en económico-empresariales } & \\
\hline & INF_ & ACT_ & C_PRO_ & C__ & CIBERACOSO \\
& EXTERNA & VIOLENCIA & SOCIALES & AGRESIVAS & \\
\hline AUTOACEPTACIÓN & -0.311 & -0.259 & 0.212 & -0.021 & -0.088 \\
& 0.000 & 0.000 & 0.003 & 0.766 & 0.217 \\
RE_POST & 199.000 & 199.000 & 199.000 & 199.000 & 199.000 \\
& 0.372 & 0.273 & -0.168 & 0.035 & 0.154 \\
AUTONOMÍA & 0.000 & 0.000 & 0.017 & 0.626 & 0.029 \\
& 200.000 & 200.000 & 200.000 & 200.000 & 200.000 \\
& 0.125 & 0.255 & -0.162 & 0.007 & 0.057 \\
DOM_ENTORNO & 0.079 & 0.000 & 0.022 & 0.925 & 0.424 \\
& 200.000 & 200.000 & 200.000 & 200.000 & 200.000 \\
& -0.250 & -0.341 & 0.148 & -0.024 & -0.117 \\
PROPÓSITO_VIDA & 0.000 & 0.000 & 0.037 & 0.741 & 0.099 \\
& 200.000 & 200.000 & 200.000 & 200.000 & 200.000 \\
& -0.359 & -0.190 & 0.210 & 0.050 & -0.036 \\
& 0.000 & 0.007 & 0.003 & 0.485 & 0.609
\end{tabular}




\section{CONCLUSIONES}

1. De acuerdo a las características de la agresión entre pares, no se evidencia relación en función a la edad; mientras que en función al sexo se encuentra que predomina la agresividad en el género masculino. Asimismo, se tiene que en el área profesional de ciencias de la salud predominan las conductas pro sociales (solidarias), en el área profesional de las ingenierías predomina el acoso por medios virtuales; mientras que con las subescalas de influencia externa, actitud hacia la violencia y conductas agresivas no existe relación.

2. En cuanto al bienestar psicológico, se concluye que a mayor edad, mayor autonomía para la toma de decisiones; y que existe más claridad en el género femenino que el masculino para la definición de sus objetivos y metas personales. En base a las áreas profesionales, el área de ciencias de la salud se caracteriza por una aceptación de los diferentes aspectos de su personalidad, buen manejo de las exigencias y oportunidades del medio, aprovechamiento de sus potencialidades y la claridad en los objetivos personales; mientras que las áreas económico-empresariales se caracterizan por la calidad de las relaciones que mantienen con sus pares y la capacidad para la toma de decisiones de manera independiente, a diferencia de las otras áreas profesionales.

3. Al relacionar las áreas de la agresión entre pares y el bienestar psicológico, se encontró que las conductas solidarias se vinculan con la aceptación de sí mismos, el aprovechamiento de sus capacidades y la claridad en las metas personales; por el contrario, la actitud favorable hacia las conductas agresivas se vincula con una baja aceptación de sí mismo y un inadecuado manejo de las oportunidades que ofrece el medio.

4. Con respecto a la edad, los adolescentes y jóvenes se caracterizan por la presencia de un vínculo entre las conductas prosociales y la influencia externa con todas las áreas del bienestar psicológico; a diferencia de los adultos, en los que la mayoría de las sub escalas de la agresión entre pares se vinculan con las áreas del bienestar psicológico. Por otro lado, se evidencia que en el género femenino el bienestar psicológico se ve afectado en mayor medida por la agresión que se da entre pares, en comparación con el género masculino.

5. En cuanto a las áreas profesionales, se encontraron datos diversos según las especialidades: En las ciencias de la salud, resulta destacable el papel que desempeñan las conductas solidarias en la forma de cómo mantener un apropiado bienestar psicológico; similar al caso de las ingenierías, en las cuales el bienestar psicológico se ve fuertemente influenciado por las conductas solidarias, expresadas como rechazo ante las conductas agresivas de los pares; y en las Económico-Empresariales, donde la influencia sobre conductas agresivas en los pares juega un papel preponderante en la determinación de un bienestar psicológico óptimo.

6. Por otro lado, se tiene que en las ciencias sociales, la agresión que se da entre pares afecta a un número más amplio de aspectos del bienestar psicológico que lo encontrado en otras áreas profesionales. Sin embargo, en las ciencias básicas, si bien no se trastocan todos los aspectos de la agresión dada entre los pares, sí hay una considerable afectación 
de dichos aspectos sobre la sensación de bienestar; mientras que en humanidades, el acoso por medios virtuales afecta en mayor medida al bienestar psicológico.

7. Finalmente, el desarrollo de la presente investigación permite contar con dos instrumentos (Escala de Agresión entre Pares de Espelage \& Holt, y Escala de Bienestar Psicológico de Ryff) válidos y confiables para ser utilizados en la realidad estudiantil limeña.

\section{REFERENCIAS BIBLIOGRÁFICAS}

Cajigas, N., Kahan, M., Luzardo, M., Najson, S., Ugo, M. C. y Zamalvide, G (2004). Escala de agresión entre pares para adolescentes y principales resultados. Acción Psicológica, 3(3), 173-186.

Castrillon, F. y Vieco, D. (2002). Actitudes justificativas del comportamiento agresivo y violento en estudiantes universitarios de Medellín. Revista de la Facultad Nacional de Salud Pública. Universidad de Antioquia, 20(2).51-66.

Diener, E., Suh, E. M., Lucas, R. E., \& Smith, H. L. (1999). Subjective well-being: Three decades of progress. Psychological Bulletin, 125, 276-302.

Espelage, D. \& Holt, M (2001). Bullying and victimización during carly adolescence: Peer influrnces and psychosocial correlates, In R. Geffner, M. Loring. \& C. Young (eds.), Bullying behavior: Current issues, research and interventions (pp. 123-142).

Espelaje, D., Mebane, S.\& Swearer, R. (2004). Gender differences in bullying: Mobing beyond mean level differences. In D. Espelaje \& Swearer (Eds.). Bullying in American schools: A social-ecological perspective on prevention and intervention (pp. 37-61) Mahwath, NJ: Eribaum.

Marqueza. M.C. (2005). Preocupaciones vitales en estudiante universitario relacionado con el bienestar psicológico y modalidades de afrontamiento. Fundamentos en Humanidades, VI(12), 143-153.

Ministerio de Educación del Perú (2010). Acoso y agresión entre estudiantes (Bullying). Lima: MINEDU.

Paredes, M.T., Álvarez, M.C. y Vernón, A, (2008). Estudio exploratorio sobre el fenómeno del bullying en la ciudad de Cali, Colombia. Revista Latinoamericana de CienciasSociales, 6(1), 295-317.

Quintana A., Montgomery W., Malaver C. \& Ruiz G. (2011). Estilos explicativos y Habilidades para la Gestión de negociación de conflictos en adolescentes espectadores de violencia entre pares (bullying). Revista de Investigación en Psicología. 11(2),139152.

Ryff, C. D. (1989). Happiness Is everything ori s it. Explorations on the mearning of psychological well-being. Journal of Personality \& Social Psychology, 57(6) 10691081.

Ryff C. D. \& Keyes, C. L. (1995). The structure of psychological well-being revisited. Journal of Personality \& Social Psychology, 69(4), 719-727. 
Salanova, S. y Martinez, I.M. (2005). Bienestar psicológico en estudiantes universitarios facilitadores y obstaculizadores de desempeño psicológico. Murcia: Universidad de Murcia.

Traumann (2008). Maltrato entre pares o bullying. Revista Chilena de Pediatría 79(1).

Velásquez C., Montgomery W., Montero V., Pomalaya R., Dioses A., Araki R. \& Reinoso D. (2008). Bienestar Psicológico, Asertividad y Rendimiento Académico en estudiantes Universitarios Sanmarquinos. Revista de Investigación en Psicología. 11(2), 139-152.

Viscardi, N. (2003). Enfrentando la violencia en las escuelas: un informe de Uruguay. En UNESCO (Ed.), Brasil: Violencia Escola: América Latina e Caribe (pp. 153-205). Brasilia: Ediciones UNESCO.

Wolff, E. (2008). Bullying, una mirada desde la salud pública. Revista Chilena de Salud Pública, vol. 12 (3). 